\title{
Use of Ring-Repeller, Double-Skimmer Electrodes for Efficient Ion Focusing in Mass Spectrometry
}

\author{
Junichi Matsumoto, Genta SaIto, and Totaro ImasaKa ${ }^{\dagger}$ \\ Department of Applied Chemistry, Graduate School of Engineering, Kyushu University, Hakozaki, Higashi, \\ Fukuoka 812-8581, Japan
}

\begin{abstract}
The design and construction of a new type of time-of-flight mass spectrometer is described. The instrument was designed to improve the ionization efficiency and to efficiently detect ions. The system performance was evaluated using the SIMION software, and was compared with the experimental results. The shapes of the repeller, extraction, and ground electrodes had a strong effect on the trajectory of ions in the processes of ion acceleration and focusing. The major difference between the theory and the experiment can be attributed to space-charge effects.
\end{abstract}

(Received January 9, 2002; Accepted February 25, 2002)

\section{Introduction}

Supersonic jet (SSJ) spectrometry has a high selectivity, which is provided by the cooling of molecules and subsequent spectral-narrowing. This spectrometric method is one of the potential candidates for the on-line real-time monitoring of polychlorinated dibenzo- $p$-dioxins (PCDDs) and dibenzofurans (PCDFs). ${ }^{1,2}$ However, the sensitivity obtained even by a stateof-the-art supersonic jet spectrometer is insufficient, since the ionization efficiency is rather low for PCDDs and PCDFs. This is the result of an efficient intersystem crossing, since the benzene rings contain numerous chlorine atoms. It is known that a laser, whose pulsewidth is shorter than the lifetime of the analyte molecule, could provide a substantial improvement in the ionization efficiency. ${ }^{3}$ This fact has been confirmed for various halogenated aromatic hydrocarbons; a molecule is excited and immediately ionized by succeeding photons before relaxation to other (e.g., triplet) levels or dissociation to other species (e.g., fragments). ${ }^{3-7}$ Recently, a laser having the same pulsewidth as the lifetime of the analyte molecule was reported to provide the maximum ionization efficiency in the case of resonance-enhanced multiphoton ionization (REMPI) combined with supersonic jet spectrometry. ${ }^{8}$ The lifetimes of PCDDs/PCDFs have not yet been reported, but are estimated to be on the order of picoseconds. A picosecond laser having a transform-limited spectral-bandwidth has been developed for this purpose. ${ }^{9}$ The use of a picosecond laser is one approach to enhance the ionization efficiency. However, further improvements in the sensitivity will be necessary for achieving on-line real-time monitoring of PCDDs/PCDFs using SSJ/REMPI/MS.

In this study, a new type of electrode assembly was designed and constructed to improve the sensitivity in SSJ/REMPI/MS. The technique described herein is essentially based on proximity ionization, which is a technique for ionizing an analyte behind the nozzle for expansion of the molecule into a

$\dagger$ To whom correspondence should be addressed.

E-mail: imasaka@cstf.kyushu-u.ac.jp vacuum. ${ }^{10}$ This approach increases the density of the analyte molecule in the ionization region, thereby improving the ionization efficiency. A variety of electrode assemblies were made and evaluated for optimizing the ion trajectory using the SIMION software. The performance of the mass spectrometer constructed in this study was examined experimentally and compared with data obtained by a computer simulation. The detection limits for phenol, chlorobenzene, and dichlorobenzene were experimentally measured, in order to evaluate the sensitivity of the mass spectrometer.

\section{Experimental}

Figure 1 shows a schematic diagram of the supersonic jet/timeof-flight mass spectrometer developed in this study. The instrument is based on the design of proximity ionization. The basic principle has been reported elsewhere, and is only briefly described here. ${ }^{10}$ The sample gas at a specified concentration is generated using a standard gas generator (Gastec, Permeater). The sample gas is introduced from a pulsed nozzle into a vacuum for mass spectrometry. The analyte molecules are

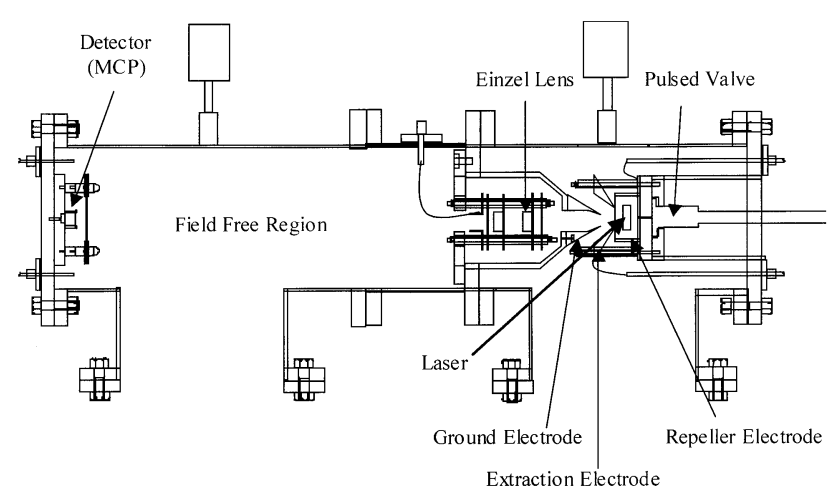

Fig. 1 New type of mass spectrometer consisting of an assembly of ring-repeller, double-skimmer electrodes. 


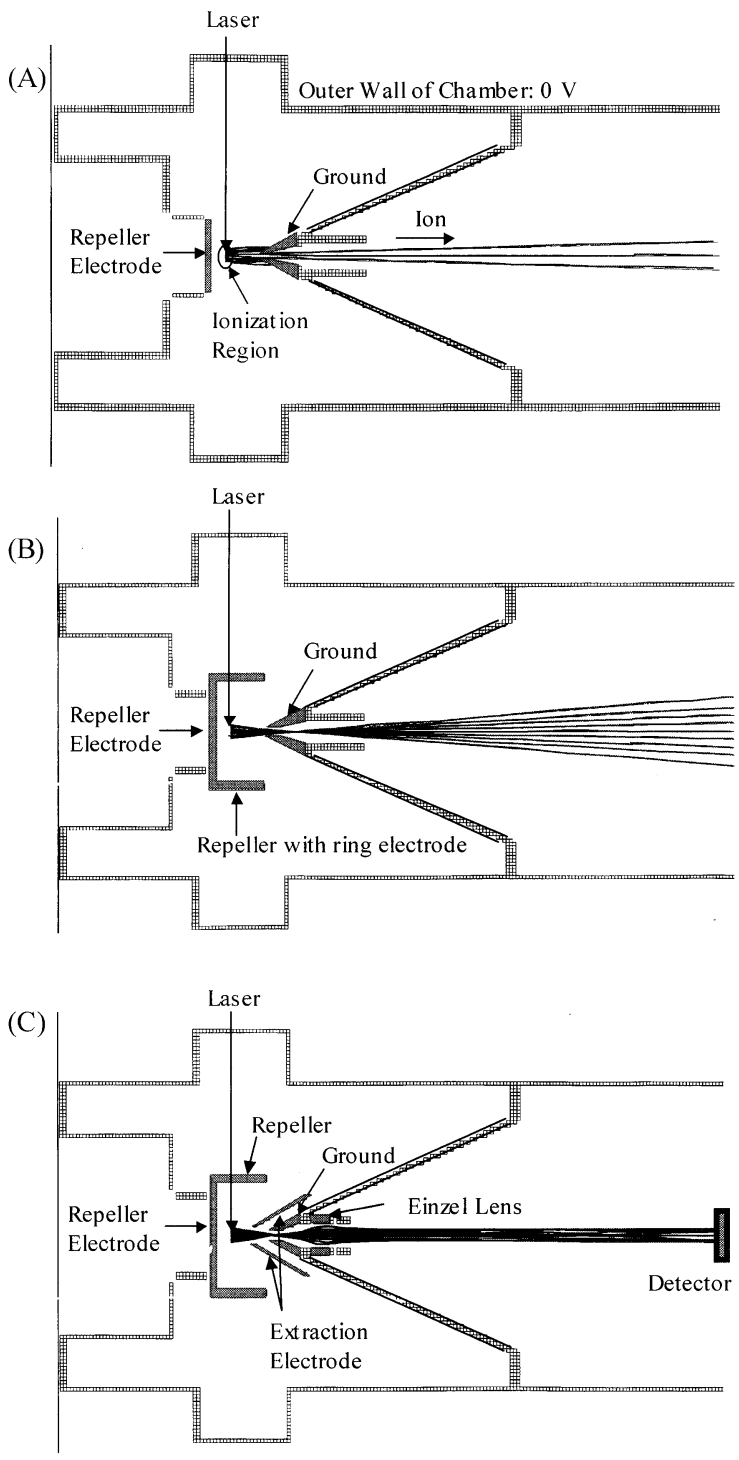

Fig. 2 Ion trajectory, as calculated by the SIMION software program. (A) Flat-repeller and ground skimmer electrodes, (B) repeller with ring electrode and ground skimmer electrode, (C) ringrepeller double-skimmer electrodes.

ionized by means of a picosecond pulsed laser. Details of the picosecond laser will be reported elsewhere. ${ }^{9}$ The mass spectrum is recorded using a digital oscilloscope. The electric potentials of the repeller, extraction, and einzel lens electrodes were changed and optimized in those experiments.

\section{Results and Discussion}

In order to avoid undesirable effects which arise via ionmolecule collision, the vacuum chamber should be evacuated sufficiently. Because of this, the vacuum chamber was separated into two parts, i.e., the ionization region and the flight region. The efficiency of ion detection is affected by the shape and diameter of the skimmer which separates these regions. In most cases, plate electrodes with mesh grids were employed for ion acceleration toward the detector. However, an assembly of plate electrodes prevents smooth jet expansion and leads to poor ion transmission efficiency due to interference by the mesh grids. It should be noted in this respect that Oser et al. used a

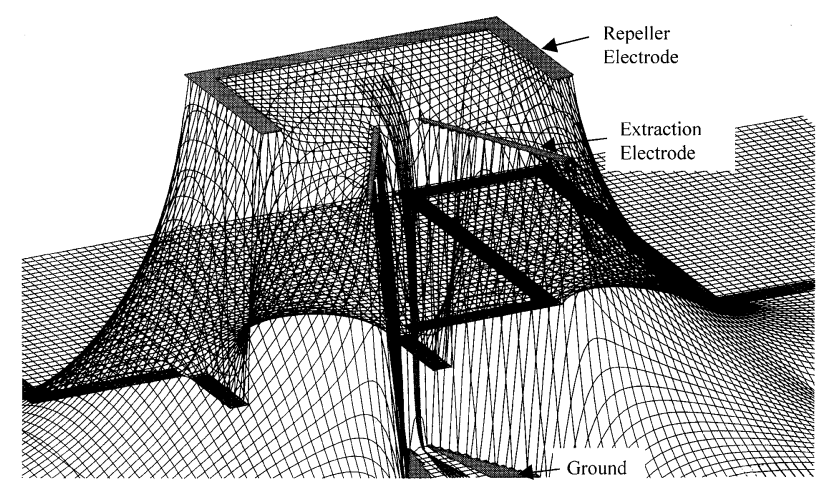

Fig. 3 Electric potential generated by ring-repeller and doubleskimmer electrodes.

skimmer for the separation of ionization and ion-acceleration regions. ${ }^{11}$

In the proximity ionization scheme, the laser beam is proposed to be line-focused in a jet to form a disk-like ionization region, which is suitable for improving both the ionization efficiency and the mass resolution. A new type of electrode assembly is desirable to properly focus the ion spread in the disk-like volume and to avoid the introduction of neutral expanded gas into the drift region. Therefore, the ion trajectory was calculated for various types of electrode assemblies by a computer simulation.

\section{Repeller and ground skimmer electrodes}

Figure 2(A) shows the ion trajectory obtained by a geometry using the repeller and ground skimmer electrodes. The electric potential of the repeller electrode was adjusted to $2000 \mathrm{~V}$. In this case, only the ion formed in the center portion can be transmitted through the ground skimmer electrode. It should be noted that a portion of the ions is accelerated toward the chamber surface, since the potential of the chamber is $0 \mathrm{~V}$. Oser and co-workers applied $-2000 \mathrm{~V}$ to the skimmer electrode, and, under these conditions, the ions were found to be efficiently extracted into the skimmer. ${ }^{11}$ In this case, the potential of the flight tube, unfortunately, becomes $-2000 \mathrm{~V}$, since the difference in the potential between the skimmer and the drift tube should be zero.

\section{Ring-repeller and skimmer electrodes}

A gradient in the electric potential toward the chamber surface was unavoidable in the previous configuration. In order to solve this problem, the ionization region was surrounded by a ring-shape repeller electrode. Figure 2(B) shows the ion trajectory obtained by the combination of a ring repeller electrode and a ground skimmer electrode. All of the ions pass through the center hole of the skimmer. However, the ion focusing point is located after (inside) the skimmer, even when the electric potential of the repeller electrode is altered over a wide voltage range. This is caused by the fact that the ion is focused and accelerated by a single gradient of electric potential, as discussed in the following section.

\section{Ring-repeller, double-skimmer electrodes}

Figure 2(C) shows the configuration of the ring-repeller, double-skimmer electrodes. The ion focus point could easily be adjusted by changing the potential applied to the extraction electrode. Therefore, the ion focus position can be changed to the center of the skimmer hole, and the diameter of the skimmer 


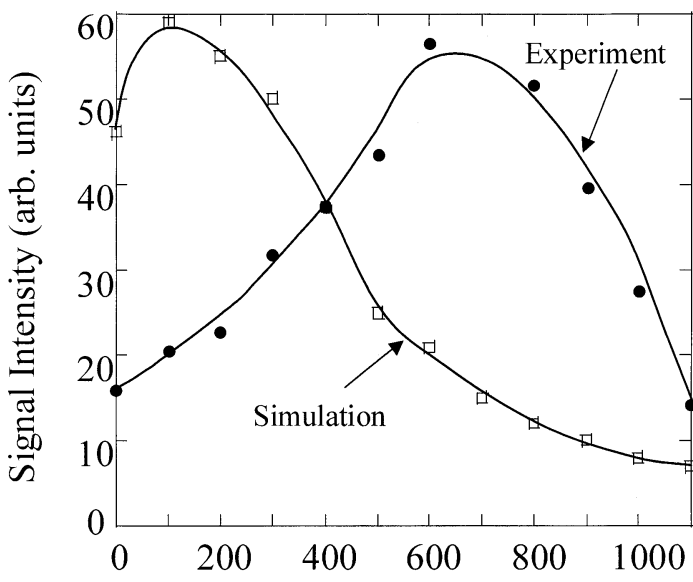

Electric Potential (V)

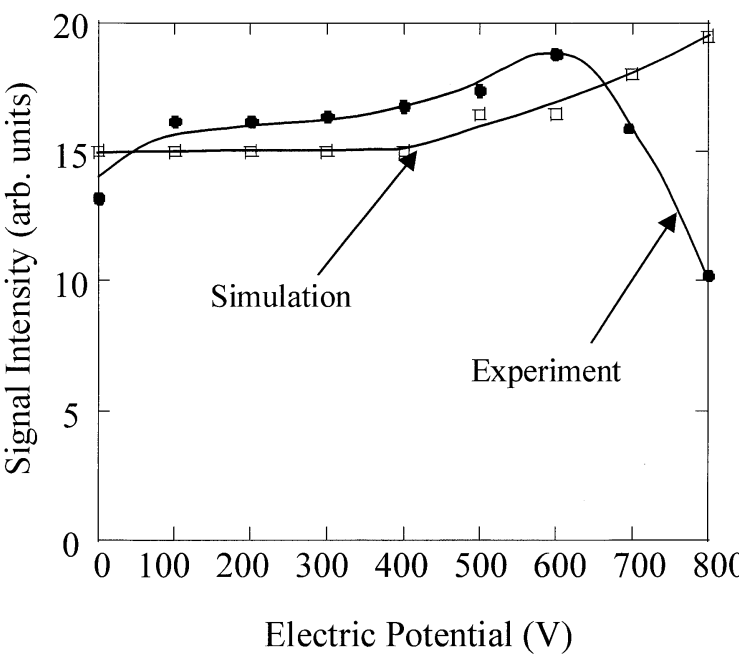

Fig. 6 Dependence of the electric potential applied to the einzel lens on the ion signal intensity.

potentials applied to the repeller and einzel lens electrodes were fixed at $1200 \mathrm{~V}$ and $0 \mathrm{~V}$, respectively. As expected, the ion signal was strongly affected by the potential of the extraction electrode in the cases of both the experiment and computer simulation. However, the maximum signal was obtained at 600 $-900 \mathrm{~V}$ in the experiment, while it shifted to $0-300 \mathrm{~V}$ in the computer simulation. This difference may arise from spacecharge effects, i.e., the expansion of ions by the repulsive force of each ion. This effect can be explained as follows. The result for a repeller potential of $1200 \mathrm{~V}$ and an extraction potential of $600 \mathrm{~V}$ is shown in Fig. 5. In this case, the ions are initially more tightly focused and then strongly dispersed. This is the reason for why the ion signal decreases at high repeller potentials in the computer simulation. However, due to the space-charge effects, the ions are more loosely focused and weakly dispersed, and, therefore, the signal increases in the experiment at high repeller potentials (e.g. $600 \mathrm{~V})$.

Figure 6 shows the relationship between the signal intensity and the electric potential applied to the einzel lens. The potentials of the repeller and extraction electrodes were adjusted to $1200 \mathrm{~V}$ and $900 \mathrm{~V}$, respectively, in this experiment. The ion signal did not change significantly even when the potential of the einzel lens was widely changed in both the experiment and computer simulation. However, it should be noted that the signal intensity rapidly decreased in the experiment at high potentials, although the theoretical value continuously increased to $800 \mathrm{~V}$. It is known that an einzel lens produces a potential barrier to focusing the ion toward the center line and this functions as an ion focusing lens. In this process, the ion is decelerated and then accelerated. A low-speed ion is easily deflected by a slight inhomogeneity in the potential, which may reduce the signal intensity substantially in the experiment. In addition, space-charge effects and molecular collisions, which are not taken into account in the computer simulation, may also deflect the ion. Thus, these unexpected effects could induce the observed difference between the experiment and the computer simulation.

\section{Mass resolution}

Figure 7 shows the relationship between mass resolution and the potential of the extraction electrode. The potential of the repeller electrode was adjusted to $1200 \mathrm{~V}$ in this experiment.
Figure 4 shows the effect of the electric potential applied to the extraction electrode on the ion signal intensity. The 


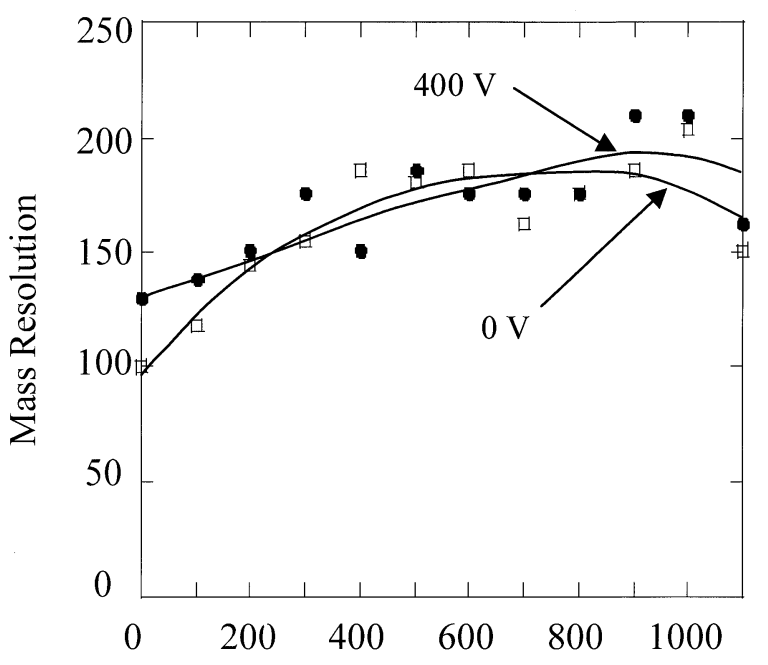

Electric Potential (V)

Fig. 7 Dependence of the electric potential applied to the extraction electrode on mass resolution. The potential applied to the einzel lens is either $400 \mathrm{~V}$ or $0 \mathrm{~V}$.

The optimum mass resolution is located at high electric potentials, which is typical for the dual-stage acceleration of ions in a time-of-flight mass spectrometer.

\section{Detection limits}

Figure 8 shows a typical mass spectrum of phenol. The sample gas, containing 5-ppm of phenol, was used in this experiment. The detection limit was calculated to be $0.6 \mathrm{ppb}$, at which the signal-to-noise $(S / N)$ ratio was 3 . The detection limits were $4.6 \mathrm{ppb}$ and $62 \mathrm{ppb}$ for chlorobenzene and dichlorobenzene, respectively. The detection limit changes, depending on the sample molecule, due to differences in the ionization efficiency. For the detection of samples in REMPI, a molecule must be excited and subsequently ionized before relaxation to intermediate states. The lifetime of the excited state of dichlorobenzene is shorter than that of phenol due to the "heavy atom effect". Therefore, the detection sensitivity is poorer for dichlorobenzene, because of the larger number of chlorine atoms that are attached to the benzene ring, resulting in a shorter lifetime of the molecule. The sensitivity reported in this work is not necessarily surprising. This is, in main part, due to the small pulse energy of the picosecond laser used in this study. It should be noted that some part of the limited sensitivity may arise from the space-charge effects. For a further discussion of the sensitivity, it may be necessary to perform the experiment at lower sample concentrations. However, this was difficult in the present study, because of the limitation in the sample preparation.

\section{Conclusions}

A new type of time-of-flight mass spectrometer was constructed and evaluated in this study. The ion signal was dependent on the shape and the electric potentials applied to the repeller, the extraction electrode, and the einzel lens. As expected, the mass resolution was also affected by the electric potential of the extraction electrode. The optimum potential for the extraction electrode was shifted to high potentials in comparison with the

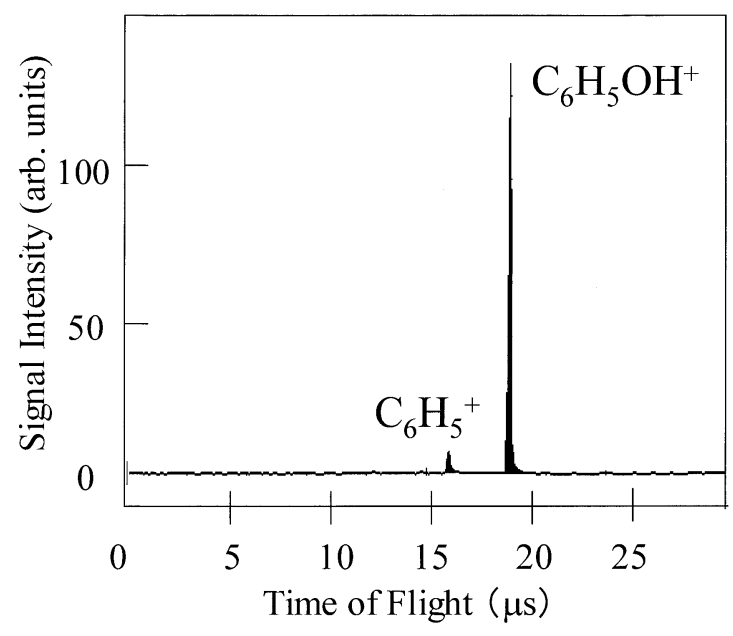

Fig. 8 Mass spectrum of phenol $(5 \mathrm{ppm})$. Potential: repeller electrode, $1200 \mathrm{~V}$; extraction electrode, $600 \mathrm{~V}$; einzel lens, $100 \mathrm{~V}$.

results of a computer simulation. This can be explained by space-charge effects. The detection limit was $0.6 \mathrm{ppb}$ for phenol, which may be substantially improved by increasing the pulse energy of the picosecond laser. These results suggest that the new type of electrode assembly described here would be useful for improving the sensitivity in REMPI/MS.

\section{Acknowledgements}

This work is supported by Grants-in-Aid for Scientific Research from the Ministry of Education, Science, Sports, and Culture of Japan and by New Energy and Industrial Technology Development Organization (NEDO) in Japan.

\section{References}

1. H. Oser, K. Copic, M. J. Coggiola, G. W. Faris, and D. R. Crosley, Chemosphere, 2001, 43, 469.

2. R. Zimmermann, H. J. Heger, A. Kettrup, and U. Boesl, Rapid Commun. Mass Spectrom., 1997, 11, 1095.

3. J. Matsumoto, C. H. Lin, and T. Imasaka, Anal. Chem., 1997, 69, 4524.

4. C. W. Wilkerson, Jr. and J. P. Reilly, Anal. Chem., 1990, 62, 1804.

5. A. Gedanken, M. B. Robin, and N. A. Kuebler, J. Phys. Chem., 1982, 86, 4096.

6. W. Dietz, H. J. Neusser, U. Boesl, E. W. Schlag, and S. H. Lin, Chem. Phys., 1982, 66, 105.

7. D. A. Gobeli, J. J. Yang, and M. A. El-Sayed, Chem. Rev., $\mathbf{1 9 8 5}, 85,529$.

8. J. Matsumoto and T. Imasaka, Anal. Chem., 1999, 71, 3763.

9. T. Deguchi, N. Takeyasu, and T. Imasaka, Rev. Sci. Instrum., accepted.

10. T. Onoda, G. Saito, and T. Imasaka, Anal. Chim. Acta, 2000, 412, 213.

11. H. Oser, R. Thanner, and H. H. Grotheer, Combust. Sci. Tech., 1996, 117, 567.

12. R. J. Cotter, "Time-of-Flight Mass Spectrometry: Instrumentation and Applications in Biological Research", 1997, American Chemical Society, Washington, D.C., 29. 